\title{
Interview
}

\section{Pflege 2018: Plattform für anregende Debatten}

\author{
Gemeinsam in die Zukunft - wohin geht die Reise in der Gesundheitspolitik? \\ Das ist Thema einer Podiumsdiskussion bei der Eröffnung des Kongresses \\ Pflege 2018 am 19. Januar, veranstaltet von Springer Pflege. Mit dabei: Jana \\ Luntz, Pflegedirektorin des Universitätsklinikums Carl Gustav Carus Dresden.
}

? Welche Erwartungen haben Sie an die Gesundheits- und Pflegepolitik, insbesondere aus der Sicht eines Uniklinikums? Was sind die drängendsten Probleme? Luntz: Während des Wahlkampfes 2017 wurde viel über den sogenannten Pflegenotstand und die schlechte Personalausstattung im Pflege- und Gesundheitssektor berichtet. Die in den Wahlprogrammen versprochenen Inhalte müssen nun sondiert und konstruktiv umgesetzt werden. Die Situation der beruflich Pflegenden muss verbessert werden. Dafür sind mehr Geld für die berufliche Ausbildung, bessere Vergütung der professionell Pflegenden und eine an den Versorgungsbedarf angepasste Personalausstattung notwendig. Um dem Pflegefachkräftemangel entgegenwirken zu können, bedarf es ganzheitlicher und strukturierter Konzepte. Es müssen zeitgemäße Aus- und Weiterbildungsmöglichkeiten geschaffen werden. Von dem politischen Diskurs erwarte ich auch, dass der Pflegeberuf mit all seinen Facetten positiver und professioneller dargestellt und die Wertigkeit dieses Berufes für die Gesellschaft herausgearbeitet wird.

? Gerade in der Hochleistungsmedizin spielt die Pflege stationär und ambulant behandelter Patienten eine wichtige Rolle. Trotzdem stehen häufig die ärztlichen Spezialisten im Fokus der Öffentlichkeit. Um dieses Ungleichgewicht zu korrigieren, gab es in Ihrem Haus eine Kampagne „Ich pflege“. Was hat sie gebracht? Luntz: Mit der Plakatkampagne wollten wir die Arbeit von insgesamt 1.870 Gesundheitsund Krankenpflegern am Universitätsklinikum Dresden würdigen und stärker ins öffentliche Bewusstsein rücken. Dabei war es uns wichtig, auf den Plakaten nicht nur ein authentisches Bild des Pflegeberufs zu vermitteln, sondern den Pflegenden selbst die Gelegenheit zu geben, etwas über ihre Motivation für den Beruf zu erzählen. Wir haben dabei auf Personen unterschiedlichen Alters gesetzt und wollten zeigen, wie vielfältig die Pflege ist. Professionelle Pflege bedeutet weitaus mehr als die Ausführung der Grundkrankenpflege. Die pflegerischen Tätigkeiten sind heutzutage sehr differenziert und hoch spezialisiert. Die positiven Rückmeldungen zur Kampagne - von den Pflegekräften selbst, Klinikdirektoren und Medizinern, aber auch von externen Entscheidungsträgern - haben uns sehr gefreut. Sie bestärken uns auf unserem Weg, die öffentliche Wahrnehmung der Pflege weiter zu fördern.

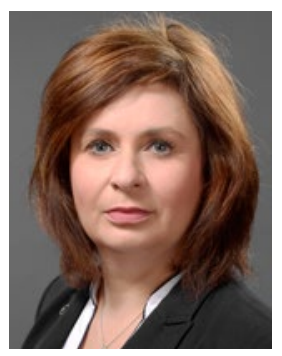

\section{Jana Luntz}

Pflegedirektorin des Universitätsklinikums Carl Gustav Carus Dresden

? Der Kongress in Berlin ist der Start ins Fortbildungsjahr 2018. Was erhoffen Sie sich von diesem Treffen der Pflegeszene? Luntz: Der Kongress bietet jedes Jahr die Möglichkeit, mit allen Partnern im Gesundheitswesen einen fach- und berufsgruppenübergreifenden Dialog zu führen. Er ist eine gute Plattform für anregende und konstruktive Diskussionen. Ich freue mich daher auf spannende Tage.

Das Interview führte Ute Burtke.

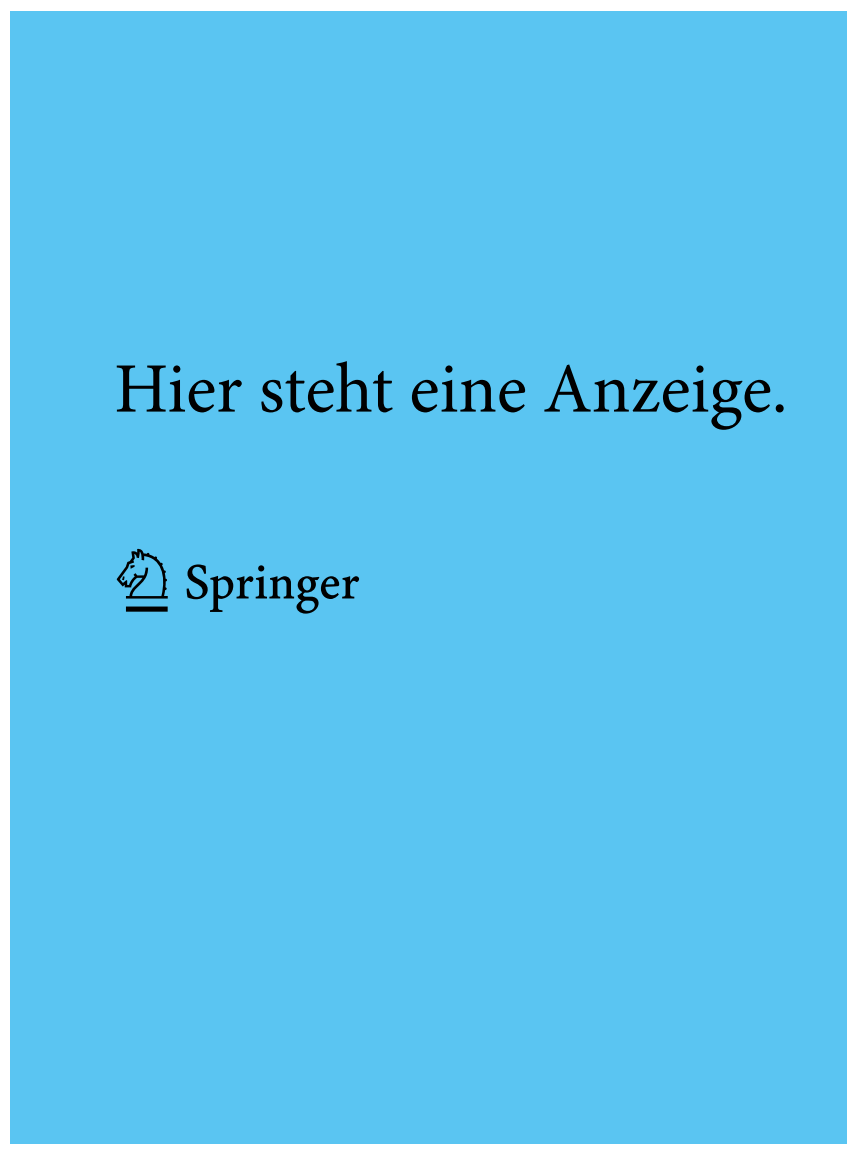

